Elżbieta Dzimida-Chmielewska $\oplus^{(1)}$ Jerzy A. Przeszowski

\title{
Physical and Nonphysical Modes in the Light Front Formulation for the LC Gauge and the Lorentz Gauge Conditions
}

Received: 14 November 2016 / Accepted: 7 March 2017 / Published online: 23 March 2017

(C) The Author(s) 2017. This article is an open access publication

\begin{abstract}
The models with free vector fields are analyzed on the light-front hypersurface. The massive vector model - Proca model — can be quantized unambiguously within the novel LF procedure, though it leads to singular terms in $x^{+}$coordinate. Also the modified models, where the Lorentz condition and the LC gauge conditions are explicitly induced by the Lagrange multipliers, are discussed. Possible massless limits are studied for all cases and modes are classified as either physical or nonphysical. A practical definition of physical modes by properties of the 1-particle states is proposed.
\end{abstract}

\section{Introduction}

There is a strange idea that for the LF quantization of QED and QCD in the LC gauge $A_{-}=0$ one needs to implement additionally the Lorentz condition $\partial^{\mu} A_{\mu}=0$, which means two simultaneous gauge fixing conditions. The outcome of this approach is the Feynman propagator in the momentum representation with three different terms [1]. We would like to use the novel LF quantization procedure, which has been recently proposed for scalar field [2], to check this situation for vector gauge fields. Unfortunately it is quite difficult to study the LC gauge $A_{-}=0$ directly, therefore here we make a preliminary analysis with the massive vector field models.

\section{Massive Vector Fields: Proca Theory}

We begin our analysis with the Proca model [3], where the Lagrangian density is

$$
\mathscr{L}_{\text {Proca }}=-\frac{1}{4} B_{\mu \nu} B^{\mu \nu}+\frac{m^{2}}{2} B^{2}, \quad B_{\mu \nu}=\partial_{\mu} B_{\nu}-\partial_{\nu} B_{\mu}
$$

and $m$ is the mass of all vector field components. This model can be quantized on the equal-time hypersurface and the vacuum expectation value of fields at arbitrary points [4] is

$$
\left\langle 0\left|\left[B_{\mu}(x), B_{\nu}(y)\right]\right| 0\right\rangle=i\left(g_{\mu \nu}+1 / m^{2} \partial_{\mu} \partial_{\nu}\right) \Delta(x-y)
$$

This article belongs to the Topical Collection "Light Cone 2016".

E. Dzimida-Chmielewska ( $\varangle)$. J. A. Przeszowski

Faculty of Physics, University of Białystok, ul. Ciołkowskiego 4L, 15-424 Białystok, Poland

E-mail: edzimida@uwb.edu.pl

J. A. Przeszowski

E-mail: j.przeszowski@uwb.edu.pl 
with $\Delta(x-y)$ being the Pauli-Villars function for a massive scalar field. We expect that the above commutator function should appear also in the LF procedure. However the terms with $\partial_{+} \Delta(x-y)$ and $\partial_{+}^{2} \Delta(x-y)$ have no limit at the LF hypersurface, as a result of the Lorentz symmetry [5]. Accordingly one cannot define the LF classical Proca field theory at the LF hypersurface. Thus the canonical LF quantization procedure [6,7], which is based on the Dirac procedure for constrained systems [8,9], cannot be applied here [10]. Fortunately the novel LF quantization procedure [2] omits these difficulties and beneath we will implement it without going into many technical details, which will be presented elsewhere.

We start our analysis with the Proca Lagrangian density (1) rewritten explicitly in the LC coordinates and components as

$$
\mathcal{L}=\frac{1}{2}\left(\partial_{+} B_{-}-\partial_{-} B_{+}\right)^{2}-\frac{1}{4}\left(\partial_{i} B_{j}-\partial_{j} B_{i}\right)^{2}+\left(\partial_{+} B_{i}-\partial_{i} B_{+}\right)\left(\partial_{-} B_{i}-\partial_{i} B_{-}\right)+m^{2} B_{+} B_{-}-\frac{m^{2}}{2} B_{i}^{2} .
$$

It leads directly to the LF canonical conjugated momenta $\pi^{+}=0, \pi^{-}=\partial_{+} B_{-}-\partial_{-} B_{+}, \pi^{i}=\partial_{-} B_{i}-\partial_{i} B_{-}$. Usually one argues that there are two primary constraints $\pi^{+} \approx 0$ and $\pi^{i}-B_{i}+\partial_{i} B_{-} \approx 0$, therefore one needs to take the Dirac procedure [8,9]. However in the novel LF formulation one does not introduce such classification of constraints and takes all non-vanishing canonical momenta $\pi^{i}$ and $\pi^{-}$for building the component $T^{++}$of the canonical energy-momentum tensor

$$
T_{\text {can }}^{++}=\pi^{-} \partial_{-} B_{-}+\pi^{i} \partial_{-} B_{i}=\pi^{-} \partial_{-} B_{-}+\left(\partial_{-} B_{i}-\partial_{i} B_{-}\right) \partial_{-} B_{i} .
$$

Before imposing the novel quantization rules, we need to select independent fields in the LF formulation, by inspecting their equations of motion. The Euler-Lagrange equations which follow from the Lagrangian (3) are $\left(\square+m^{2}\right) B_{\mu}=\partial_{\mu}(\partial \cdot B)$, where $\square=\partial^{\mu} \partial_{\mu}=2 \partial_{+} \partial_{-}-\partial_{i}^{2}$ and $\partial \cdot B=\partial^{\mu} B_{\mu}$. However these equations can be equivalently rewritten as $\left(\square+m^{2}\right) B_{\mu}=0, \partial^{\mu} B_{\mu}=0$, thus all components $B_{\mu}$ satisfy the Klein-Gordon equation of motion, though only three of them are independent modes due to the Lorentz condition. Seemingly, $B_{-}$should be taken as one of them, since its conjugated canonical momentum $\pi^{-}=\partial_{+} B_{-}-\partial_{-} B_{+}$, contains temporal derivative (velocity). However the closer examination of the Euler-Lagrange shows that there are two constraint equations

$$
\left(\Delta_{\perp}-m^{2}\right) B_{-}=\partial_{-}\left(\pi^{-}+\partial_{i} B_{i}\right), \quad 2 \partial_{-} B_{+}=\partial_{i} B_{i}-\pi^{-}
$$

Since the partial differential operator $\left(\Delta_{\perp}-m^{2}\right)$ is invertible, then $B_{-}$can be unambiguously determined in terms of $\pi^{-}$and $B_{i}$ fields. Further for avoiding the non-local terms we may introduce new fields $\Sigma$ and $C_{i}$ by the differential equations $\left(\Delta_{\perp}-m^{2}\right) \Sigma=\left(\pi^{-}+\partial_{i} B_{i}\right)$ and $C_{i}=A_{i}-\partial_{i} \Sigma$, respectively. The best choice of three independent fields is $\Sigma$ and $C_{i}$, which satisfy the Klein-Gordon equations $\left(2 \partial_{+} \partial_{-}-\Delta_{\perp}+m^{2}\right) \Sigma=$ $0,\left(2 \partial_{+} \partial_{-}-\Delta_{\perp}+m^{2}\right) C_{i}=0$. The constraint equations

$$
2 \partial_{-} B_{+}=2 \partial_{i} C_{i}+\left(\Delta_{\perp}+m^{2}\right) \Sigma, \quad B_{i}=C_{i}+\partial_{i} \Sigma, \quad B_{-}=\partial_{-} \Sigma,
$$

express $B_{\mu}$ fields in terms of independent fields $\Sigma$ and $C_{i}$, though for $B_{+}$one needs to solve the partial differential equation. ${ }^{1}$ Then for new fields we rewrite $T_{\text {can }}^{++}$as $T_{\text {can }}^{++} \approx\left(\partial_{-} C_{i}\right)^{2}+m^{2}\left(\partial_{-} \Sigma\right)^{2}$, where, approximate equality, symbolized by a squiggly equal sign $\approx$, means that terms, which are total partial derivatives, have been omitted. The LF quantization is performed at the arbitrary fixed $x^{+}$coordinate, but for the clarity we just take $x^{+}=0$ and denote $\bar{x}=\left(x^{-}, \boldsymbol{x}_{\perp}\right)$.

We postulate as the quantization rule on the LF hypersurface $x^{+}=0$ the following relations for the independent fields $C_{i}, \Sigma$

$$
i \partial_{-} C_{i}(\bar{x})=\left[C_{i}(\bar{x}), P_{-}\right], \quad i \partial_{-} \Sigma(\bar{x})=\left[\Sigma(\bar{x}), P_{-}\right],
$$

where the generator of translation in $x^{-}$direction is defined as $P_{-}=\int_{\mathbb{R}^{3}} d^{3} \bar{x} T_{c a n}^{++}(\bar{x})$. These relations can be identically satisfied provided the following commutators hold at the LF hypersurface

$$
\left[C_{i}(\bar{x}), \partial_{-} C_{j}(\bar{y})\right]=\frac{i}{2} \delta_{i j} \delta^{3}(\bar{x}-\bar{y}), \quad\left[\Sigma(\bar{x}), \partial_{-} \Sigma(\bar{y})\right]=\frac{i}{2 m^{2}} \delta^{3}(\bar{x}-\bar{y}), \quad\left[C_{i}(\bar{x}), \partial_{-} \Sigma(\bar{y})\right]=0 .
$$

\footnotetext{
${ }^{1}$ We will not introduce the inverse operator $\left[\partial_{-}\right]^{-1}$, which needs the boundary conditions in $\pm \infty$ in $x^{-}$direction. Instead later we will solve this equation in the momentum space, which seems to be more physical.
} 
Next we take the vacuum expectation value of these commutators, with the normalized vacuum state $\langle 0 \mid 0\rangle=1$ and further we decompose them into the matrix elements. For achieving this goal we need to introduce smooth fields $C_{i}[f]$ and $\Sigma[f]$ by smearing with a real-valued test function $f(\bar{x}) \in \mathscr{S}^{\prime}\left(\mathbb{R}^{3}\right)$, where we write $C_{i}[f]=\int_{\mathbb{R}^{3}} d^{3} \bar{x} C_{i}(\bar{x}) f(\bar{x}), \Sigma[f]=\int_{\mathbb{R}^{3}} d^{3} \bar{x} \Sigma(\bar{x}) f(\bar{x}) .^{2}$

Then we introduce another LF assumption, that the smooth operators $C_{i}[f]$ and $\Sigma[f]$ when acting on the vacuum state $|0\rangle$ produce 1-particle states,

$$
C_{i}[f]|0\rangle=\int_{\mathbb{R}^{3}} d \Gamma(\bar{k}) \tilde{f}(\bar{k})\left|\bar{k}, c_{i}\right\rangle, \quad \Sigma[f]|0\rangle=\int_{\mathbb{R}^{3}} d \Gamma(\bar{k}) \tilde{f}(\bar{k})|\bar{k}, \sigma\rangle, \quad d \Gamma(\bar{k})=\frac{d^{3} \bar{k}}{(2 \pi)^{3}} \frac{\Theta\left(k^{+}\right)}{2 k^{+}},
$$

where $\tilde{f}(\bar{k})$ is the Fourier transform of $f(\bar{x})$, while $\left|\bar{k}, c_{i}\right\rangle$ and $|\bar{k}, \sigma\rangle$ are the 1-particle eigenstates of the kinematical translation generators $\bar{P}_{\mu}=\left(P_{-}, P_{i}\right){ }^{3}$ The calculation directly leads to the non-vanishing matrix elements $\langle 0|\Sigma(0)| \bar{k}, \sigma\rangle=1 / \mathrm{m}^{2}$ and $\left\langle 0\left|C_{i}(0)\right| \bar{k}, c_{j}\right\rangle=\delta_{i j}$ which further allows to determine the non-vanishing inner products for 1-particle eigenstates of kinematical momenta $\langle\bar{k}, \sigma \mid \bar{p}, \sigma\rangle=1 / m^{2} \delta_{\Gamma}(\bar{k}, \bar{p}),\left\langle\bar{k}, c_{i} \mid \bar{p}, c_{j}\right\rangle=$ $\delta_{i j} \delta_{\Gamma}(\bar{k}, \bar{p})$, where $\delta_{\Gamma}(\bar{k}, \bar{p})=(2 \pi)^{3} 2 k^{+} \delta^{2}\left(\boldsymbol{k}_{\perp}-\boldsymbol{p}_{\perp}\right) \delta\left(k^{+}-p^{+}\right)$.

Then we introduce equations for the quantum field operators in the form identical to the classical Euler-Lagrange equations, since for a free field model there is no need for the renormalization effects and counter-terms. We need to express all partial derivatives in terms of commutators with respective translation generators-for example $i \partial_{\mu} C_{i}(x)=\left[C_{i}(x), P_{\mu}\right]$. For the second order partial derivatives this leads to double commutators, which will simplify considerably if it acts on the vacuum state, for example we have, $\partial_{+} \partial_{-} C_{i}(x)=-\left[\left[C_{i}(x), P_{-}\right], P_{+}\right]$and $\partial_{+} \partial_{-} C_{i}(x)|0\rangle=-P_{-} P_{+} C_{i}(x)|0\rangle$, since $P_{\mu}|0\rangle=0$. Accordingly for the independent smooth dynamical fields $\Sigma[f]$ and $C_{i}[f]$ we obtain the following dynamical LF Schrödinger equations

$$
\left(2 P_{+} P_{-}-P_{\perp}^{2}-m^{2}\right) \Sigma[f]|0\rangle=0, \quad\left(2 P_{+} P_{-}-P_{\perp}^{2}-m^{2}\right) C_{i}[f]|0\rangle=0,
$$

which then lead to the eigenvalue equations for the LF Hamiltonian

$$
P_{+}|\bar{k}, \sigma\rangle=\frac{m^{2}+k_{\perp}^{2}}{2 k^{+}}|\bar{k}, \sigma\rangle \quad P_{+}\left|\bar{k}, c_{i}\right\rangle=\frac{m^{2}+k_{\perp}^{2}}{2 k^{+}}\left|\bar{k}, c_{i}\right\rangle, \quad k^{+}>0 .
$$

Accordingly the 1-particle states $|\bar{k}, \sigma\rangle$ and $\left|\bar{k}, c_{i}\right\rangle$ are the eigenfunctions (eigenvectors) for all components of momentum operators $P_{\mu}$. Then we may proceed analogously with other equations and we obtain the nondynamical LF Schrödinger equations

$$
\begin{aligned}
B_{-}[f]|0\rangle & =i P_{-} \Sigma[f]|0\rangle, \quad B_{j}[f]|0\rangle=C_{j}[f]|0\rangle+i P_{j} \Sigma[f]|0\rangle, \\
P_{-} B_{+}[f]|0\rangle & =P_{j} C_{j}[f]|0\rangle+i \frac{P_{\perp}^{2}-m^{2}}{2} \Sigma[f]|0\rangle .
\end{aligned}
$$

Equation (12b) can be transformed, using the Klein-Gordon equation in the momentum space $\left(2 P_{+} P_{-}-\right.$ $\left.P_{\perp}^{2}-m^{2}\right) B_{+}[f]=0$ into a form, which unambiguously allows for a unique solution

$$
\left(P_{\perp}^{2}+m^{2}\right) B_{+}[f]|0\rangle=2 P_{+}\left(P_{j} C_{j}[f]|0\rangle+i \frac{P_{\perp}^{2}-m^{2}}{2} \Sigma[f]|0\rangle\right) .
$$

All this allows to express $B_{\mu}[f]|0\rangle$ in its generic form

$$
B_{\mu}[f]|0\rangle=\int_{\mathbb{R}^{3}} d \Gamma(\bar{k}) \tilde{f}(\bar{k}) \sum_{a=1,2,3} \epsilon_{\mu}^{a}(\bar{k})|\bar{k}, a\rangle,
$$

\footnotetext{
2 These smooth fields are very useful for inferring the commutators (8) from the quantization rules (7).

${ }^{3}$ One may derive this assumption from the nonnegative spectrum of $P_{-}=P^{+}$operator and the Lorentz symmetry of vacuum state $P_{\mu}|0\rangle=0$.
} 
where we have introduced new 1-particle eigenfunctions of 4-momenta $|\bar{k}, 1\rangle=\left|\bar{k}, c_{1}\right\rangle,|\bar{k}, 2\rangle=\left|\bar{k}, c_{2}\right\rangle$ and $|\bar{k}, 3\rangle=i m|\bar{k}, \sigma\rangle$, which have the orthonormal positive inner products $\langle\bar{p}, a \mid \bar{k}, b\rangle=\delta_{a b} \delta_{\Gamma}(\bar{p}, \bar{k}), a, b \in$ $\{1,2,3\}$. The the polarization vectors $\epsilon_{\mu}^{a}(\bar{k})$ have the form of one-column matrix ${ }^{4}$

$$
\epsilon_{\mu}^{1}(\bar{k})=\frac{1}{k^{+}}\left[\begin{array}{c}
k_{1} \\
0 \\
k^{+} \\
0
\end{array}\right], \quad \epsilon_{\mu}^{2}(\bar{k})=\frac{1}{k^{+}}\left[\begin{array}{c}
k_{2} \\
0 \\
0 \\
k^{+}
\end{array}\right], \quad \epsilon_{\mu}^{3}(\bar{k})=\frac{1}{m}\left[\begin{array}{c}
\frac{k_{\perp}^{2}-m^{2}}{2 k^{+}} \\
k^{+} \\
k_{1} \\
k_{2}
\end{array}\right] .
$$

Any 1-particle state, which is simultaneously an eigenvector of all Poincare generators $P_{\mu}$ with energy eigenvalue consistent with the Lorentz symmetry and its inner product is positively definite, describes a physical mode. Otherwise it is a nonphysical mode. Our previous analysis evidently shows that there are three physical modes in the Proca model quantized at the LF hypersurface. However the polarization vector $\epsilon_{\mu}^{3}$ contains the overall factor $1 / m$, thus it has no massless limit $m \rightarrow 0$. Therefore we cannot directly move from the massive Proca fields into the gauge fields, though we have the Lorentz condition $\partial^{\mu} B_{\mu}=0$ already for nonzero mass. For proving the consistency of our result with the usual quantization we may calculate the 2-point Wightman function (the vacuum expectation value of unordered product of two quantum field operators) for arbitrary points

$$
\left\langle 0\left|B_{\mu}(x) B_{v}(y)\right| 0\right\rangle=-\left(g_{\mu \nu}+\frac{1}{m^{2}} \partial_{\mu} \partial_{\nu}\right) \int_{\mathbb{R}^{3}} d \Gamma(\bar{k}) e^{-i \bar{k} \cdot(\bar{x}-\bar{y})} e^{-i \frac{m^{2}+k_{\perp}^{2}}{2 k^{+}}\left(x^{+}-y^{+}\right)},
$$

which agrees with the commutator function (2) since in the LF momentum representation

$$
\Delta(x)=i \int_{\mathbb{R}^{3}} d \Gamma(\bar{k})\left[e^{i \bar{k}(\bar{x}-\bar{y})} e^{i \frac{m^{2}+k_{\perp}^{2}}{2 k^{+}}\left(x^{+}-y^{+}\right)}-e^{-i \bar{k}(\bar{x}-\bar{y})} e^{-i \frac{m^{2}+k_{\perp}^{2}}{2 k^{+}}\left(x^{+}-y^{+}\right)}\right] .
$$

Thus we have been able to omit all problems which appear in the coordinate space formulation, where terms singular at $x^{+}=0$ appear.

\section{The Modified Proca Model with the Lorentz Condition}

Now we introduce a modification to the Proca model by adding the Lorentz condition $\partial \cdot B=0$, by means of the Lagrange multiplier field $\Lambda$, into the Lagrangian density (3). This will slightly change the Euler-Lagrange equations $\left(\square+m^{2}\right) B_{\mu}=\partial_{\mu} \Lambda, \partial^{\mu} B_{\mu}=0$ and the canonical momentum $\pi^{-}=\partial_{+} B_{-}-\partial_{-} B_{+}+\Lambda$. Also the equation for a new field is modified $\left(\Delta_{\perp}-m^{2}\right) \Sigma=\left(\pi^{-}+\partial_{i} B_{i}-\Lambda\right)$ and the energy-momentum tensor component is $T^{++}=\left(\partial_{-} C_{i}\right)^{2}+m^{2}\left(\partial_{-} \Sigma\right)^{2}-2 \partial_{-} \Sigma \partial_{-} \Lambda$. Then the independent fields are $C_{i}, \Sigma, \Lambda$ with dynamical equations $\left(\square+m^{2}\right) C_{i}=0,\left(\square+m^{2}\right) \Sigma=\Lambda, \square \Lambda=0$.

Following steps analogous to those from previous section, we find non-vanishing LF commutators for independent fields

$$
\begin{aligned}
{\left[C_{i}(\bar{x}), \partial_{-} C_{j}(\bar{y})\right] } & =\frac{i}{2} \delta_{i j} \delta^{3}(\bar{x}-\bar{y}), & {\left[\Lambda(\bar{x}), \partial_{-} \Lambda(\bar{y})\right] } & =-\frac{i}{2} m^{2} \delta^{3}(\bar{x}-\bar{y}), \\
{\left[\Lambda(\bar{x}), \partial_{-} \Sigma(\bar{y})\right] } & =-\frac{i}{2} \delta^{3}(\bar{x}-\bar{y}), & {\left[\Sigma(\bar{x}), \partial_{-} \Lambda(\bar{y})\right] } & =-\frac{i}{2} \delta^{3}(\bar{x}-\bar{y}) .
\end{aligned}
$$

and the 1-particle states with their non-vanishing inner products

$$
\langle\bar{k}, \lambda \mid \bar{p}, \lambda\rangle=-m^{2} \delta_{\Gamma}(\bar{k}, \bar{p}), \quad\langle\bar{k}, \sigma \mid \bar{p}, \lambda\rangle=-\delta_{\Gamma}(\bar{k}, \bar{p}), \quad\left\langle\bar{k}, c_{i} \mid \bar{p}, c_{j}\right\rangle=\delta_{i j} \delta_{\Gamma}(\bar{k}, \bar{p}),
$$

The dynamical equations give rise to the dynamical LF Schrödinger equations

$$
2 P_{+} k^{+}\left|\bar{k}, c_{i}\right\rangle=\left(k_{\perp}^{2}+m^{2}\right)\left|\bar{k}, c_{i}\right\rangle, \quad 2 P_{+} k^{+}|\bar{k}, \lambda\rangle=k_{\perp}^{2}|\bar{k}, \lambda\rangle, \quad 2 P_{+} k^{+}|\bar{k}, \sigma\rangle=\left(k_{\perp}^{2}+m^{2}\right)|\bar{k}, \sigma\rangle-|\bar{k}, \lambda\rangle,
$$

${ }^{4}$ We denote $\epsilon_{+}^{a}(\bar{k})$ in the first row, then $\epsilon_{-}^{a}(\bar{k})$ in the second row, then $\epsilon_{1}^{a}(\bar{k})$ in the third row and at last $\epsilon_{2}^{a}(\bar{k})$ in the fourth row. 
which show that the 1-particle state $|\vec{k}, \sigma\rangle$ is not an eigenfunction of the LF Hamiltonian $P_{+}$. From nondynamical equations $B_{-}=\partial_{-} \Sigma, 2 \partial_{-} B_{+}=2 \partial_{i} C_{i}+\left(\Delta_{\perp}+m^{2}\right) \Sigma-\Lambda, B_{i}=C_{i}+\partial_{i} \Sigma$, one finds the non-dynamical Schrödinger equations, which lead to

$$
\begin{aligned}
& B_{-}[f]|0\rangle=\int_{\mathbb{R}^{3}} d \Gamma(\bar{k}) \tilde{f}(\bar{k})\left(i k^{+}\right)|\bar{k}, \sigma\rangle, \quad B_{i}[f]|0\rangle=\int_{\mathbb{R}^{3}} d \Gamma(\bar{k}) \tilde{f}(\bar{k})\left(\left|\bar{k}, c_{i}\right\rangle+i k_{i}|\bar{k}, \sigma\rangle\right), \\
& B_{+}[f]|0\rangle=\int_{\mathbb{R}^{3}} d \Gamma(\bar{k}) \tilde{f}(\bar{k})\left(\frac{k_{i}}{k^{+}}\left|\bar{k}, c_{i}\right\rangle+i \frac{k_{\perp}^{2}-m^{2}}{2 k^{+}}|\bar{k}, \sigma\rangle+\frac{i}{2 k^{+}}|\bar{k}, \lambda\rangle\right) .
\end{aligned}
$$

We may define new 1-particle states $|\bar{k}, 0\rangle=\frac{i}{m}|\bar{k}, \lambda\rangle,|\bar{k}, 3\rangle=i m|\bar{k}, \sigma\rangle-\frac{i}{m}|\bar{k}, \lambda\rangle,|\bar{k}, 1\rangle=\left|\bar{k}, c_{1}\right\rangle,|\bar{k}, 2\rangle=$ $\left|\bar{k}, c_{2}\right\rangle$, which are the eigenstates of the Hamiltonian operator $P_{+}$, for $k^{+}>0$,

$$
P_{+}|\bar{k}, a\rangle=\frac{k_{\perp}^{2}+m^{2}}{2 k^{+}}|\bar{k}, a\rangle, \quad P_{+}|\bar{k}, 0\rangle=\frac{k_{\perp}^{2}}{2 k^{+}}|\bar{k}, 0\rangle,
$$

where $a \in\{1,2,3\}$. The non-vanishing inner product for these states $\langle\bar{p}, a \mid \bar{k}, b\rangle=\delta_{a b} \delta_{\Gamma}(\bar{p}, \bar{k})$ and $\langle\bar{p}, 0 \mid \bar{k}, 0\rangle=-\delta_{\Gamma}(\bar{p}, \bar{k})$. Therefore there are 3 physical modes with mass $m$, described by the 1-particle states $|\vec{k}, a\rangle$ with $a \in\{1,2,3\}$ and one unphysical massless mode, described by $|\vec{k}, 0\rangle$.

For the case $m \neq 0$ we find the smooth fields acting on the vacuum state

$$
B_{\mu}[f]|0\rangle=\int_{\mathbb{R}^{3}} d \Gamma(\bar{k}) \tilde{f}(\bar{k}) \sum_{a=1,2,3} \epsilon_{\mu}^{a}(\bar{k})|\bar{k}, a\rangle+\frac{1}{m} P_{\mu} \int_{\mathbb{R}^{3}} d \Gamma(\bar{k}) \tilde{f}(\bar{k})|\bar{k}, 0\rangle,
$$

where polarization vectors are the same as in the Proca model (14). There is a cancellation of $1 / m$ contributions between $|\bar{k}, 3\rangle$ and $|\bar{k}, 0\rangle$ states, which allows for the massless limit $m \rightarrow 0$. We denote this limit by $B_{\mu} \rightarrow A_{\mu}$ and we find

$$
A_{\mu}[f]|0\rangle=\int_{\mathbb{R}^{3}} d \Gamma(\bar{k}) \tilde{f}(\bar{k}) \sum_{a=1,2,3} \epsilon_{\mu}^{a}(\bar{k})|\bar{k}, a\rangle+P_{\mu} \int_{\mathbb{R}^{3}} d \Gamma(\bar{k}) \tilde{f}(\bar{k}) \frac{1}{k_{\perp}}|\bar{k}, 0\rangle,
$$

where now the 1-particle states are slightly redefined $|\bar{k}, 3\rangle=\frac{i}{k_{\perp}}|\bar{k}, \lambda\rangle,|\bar{k}, 0\rangle=i k_{\perp}|\bar{k}, \sigma\rangle$, with the nonvanishing inner product for them $\langle\bar{k}, 0 \mid \bar{p}, 3\rangle=\langle\bar{k}, 3 \mid \bar{p}, 0\rangle=-\delta_{\Gamma}(\bar{k}, \bar{p})$. Here we stress that $|\bar{k}, 3\rangle$ is the eigenfunction of the LF Hamiltonian $P_{+}$, while $|\bar{k}, 0\rangle$ is not

$$
P_{+}|\bar{k}, 3\rangle=\frac{k_{\perp}^{2}}{2 k^{+}}|\bar{k}, 3\rangle, \quad P_{+}|\bar{k}, 0\rangle=\frac{k_{\perp}^{2}}{2 k^{+}}|\bar{k}, 0\rangle-\frac{k_{\perp}^{2}}{2 k^{+}}|\bar{k}, 3\rangle .
$$

Therefore for the massless limit there are only 2 physical modes described by the 1-particle states $|\bar{k}, a\rangle$ with $a \in\{1,2\}$ and 2 nonphysical modes described by the 1-particle states $|\bar{k}, 0\rangle$ and $|\bar{k}, 3\rangle$. In Eq. (23) the polarization vectors are slightly modified, $\epsilon_{\mu}^{1,2}$ remain unchanged as in Eq. (14), while $\epsilon_{+}^{3}(\bar{k})=\frac{k_{\perp}}{k^{+}}, \epsilon_{-}^{3}(\bar{k})=$ $0, \epsilon_{1}^{3}(\bar{k})=0, \epsilon_{2}^{3}(\bar{k})=0$.

If one tries to impose the additional restriction for the gauge field potential $A_{-}=0$ one evidently removes the unphysical modes $|\bar{k}, 0\rangle$ and $|\bar{k}, 3\rangle$.

\section{Modified Proca Model with LC Weyl Condition}

It is very difficult to perform the LF quantization for the $\mathrm{LC}$ gauge $B_{-}=0$. Therefore we may check a simpler possibility by imposing the LC Weyl condition $B_{+}=0$. This gauge has been discussed in [11] within the DLCQ quantization and in [12] within the continuous formulation. Here we impose $B_{+}=0$ by means of the Lagrange multiplier field $\Lambda$ into the Lagrangian density (3). This leads to the Euler-Lagrange equations which are all dynamical $\partial_{+} \pi^{-}=0, \partial_{+} B_{-}=\pi^{-}+\partial_{i} B_{i}, \quad\left(2 \partial_{+} \partial_{-}-\Delta_{\perp}\right) B_{i}=\partial_{i} \pi^{-}$, while the canonical energy-momentum tensor component is $T^{++}=\left(\partial_{-} B_{i}\right)^{2}+\pi^{-} \partial_{-} B_{-}$. Following steps analogous to those in previous sections we find the commutators for independent fields are

$$
\left[B_{-}(\bar{x}), \pi^{-}(\bar{y})\right]=i \delta^{3}(\bar{x}-\bar{y}), \quad 2\left[B_{i}(\bar{x}), \partial_{-} B_{j}(\bar{y})\right]=i \delta_{i j} \delta^{3}(\bar{x}-\bar{y}),
$$


Between the 1-particle states, which appear naturally, the non-vanishing inner products $\left\langle\bar{k}, a_{-} \mid \bar{p}, \pi^{-}\right\rangle=$ $2 i k^{+} \delta_{\Gamma}(\bar{k}, \bar{p})$ and $\left\langle\bar{k}, a_{i} \mid \bar{p}, a_{j}\right\rangle=\delta_{i j} \delta_{\Gamma}(\bar{k}, \bar{p})$. After the redefinitions of 1 -particle states

$$
|\bar{k}, 3\rangle=\left|\bar{k}, a_{-}\right\rangle-\frac{2 k^{+}}{k_{\perp}^{2}+m^{2}} k_{j}\left|\bar{k}, a_{j}\right\rangle, \quad|\bar{k}, j\rangle=\left|\bar{k}, a_{j}\right\rangle-i \frac{k_{j}}{k_{\perp}^{2}+m^{2}}|\bar{k}, \pi\rangle, \quad|\bar{k}, 0\rangle=\frac{1}{2 k^{+}}\left|\bar{k}, \pi^{-}\right\rangle,
$$

we find that $|\bar{k}, i\rangle$ and $|\bar{k}, 0\rangle$ are the eigenfunctions of the LF Hamiltonian $P_{+}$, while $|\bar{k}, 3\rangle$ is not. Therefore there are 2 physical modes described by the 1-particle states $|\bar{k}, i\rangle$ with $i \in\{1,2\}$ and 2 nonphysical modes described by the 1-particle states $|\bar{k}, 3\rangle$ and $|\bar{k}, 0\rangle$. Further we find the expression

$$
B_{\mu}[f]|0\rangle=\int_{\mathbb{R}^{3}} d \Gamma(\bar{k}) \tilde{f}(\bar{k}) \sum_{a=0,1,2,3} \epsilon_{\mu}^{a}(\bar{k})\left|\bar{k}, c_{a}\right\rangle,
$$

with the polarization vectors given in (28). The massless limit $m \rightarrow 0$ can be safely taken and the conclusion that there are 2 physical modes and 2 nonphysical modes remains unchanged.

When one adds the additional condition on the gauge field $\partial^{\mu} B_{\mu}=0$, which in the momentum space takes the form $\left.P_{+} B_{[} f\right]|0\rangle=P_{i} B_{i}[f]|0\rangle$, then one removes the nonphysical modes.

\section{Conclusions and Prospects for Further Research}

In this paper we have considered the gauge field model, where a mass term is added as a regularization of the possible infra-red singularities. These massive vector fields can be quantized on the LF hypersurface within the novel quantization procedure. For the Proca model we have found 3 physical modes, all with mass $m$. Then we have introduced additional conditions on the vector fields. The Lorentz condition $\partial^{\mu} B_{\mu}=0$ leads to 3 physical modes with mass $m$ and 1 nonphysical massless modes. In the massless limit $m \rightarrow 0$, one of physical mode is no longer described by an eigenvector of the LF Hamiltonian thus we conclude that for massless fields there are 2 physical and 2 nonphysical modes, as expected. When we introduce the LF Weyl condition $B_{+}=0$, then the LF quantization leads to 2 physical modes, with mass $m$ and 2 nonphysical modes. This classification survives the massless limit.

If we add additional conditions then we see that some nonphysical modes needs to be discarded. Usually nonphysical modes are needed for reaching a smooth behavior of the Feynman propagator. But for a decisive conclusion one needs to quantize at the LF hypersurface the gauge field system with 2 conditions from the beginning. This will be analyzed in the nearest future.

\section{Appendix: Notation and Formulas}

We use the covariant notation, where the LF longitudinal coordinates are defined as $x^{ \pm}=\left(x^{0} \pm x^{3}\right) / \sqrt{2}$ and the partial derivatives are denoted as $\partial_{ \pm}=\partial / \partial x^{ \pm}$. The Minkowski space-time metric tensor has non-vanishing components $\eta_{+-}=\eta_{-+}=1, \eta_{i j}=-\delta_{i j},(i, j) \in\{1,2\}$. The summation over repeated indexes is always understood.

The massive vector field components, we denote by $B_{\mu}=\left(B_{+}, B_{-}, B_{i}\right)$ with $i \in\{1,2\}$, for keeping it distinct from the massless vector field, which is commonly described by $A_{\mu}$.

The polarization vectors for the LF Weyl gauge condition are

$$
\epsilon_{\mu}^{0}(\bar{k})=\frac{i \gamma}{k_{\perp}}\left[\begin{array}{c}
0 \\
\gamma k_{\perp} \\
k_{1} \\
k_{2}
\end{array}\right] \quad \epsilon_{\mu}^{1}(\bar{k})=\frac{1}{k_{\perp}}\left[\begin{array}{c}
0 \\
\gamma k_{1} \\
0 \\
k_{\perp} \\
0
\end{array}\right] \quad \epsilon_{\mu}^{2}(\bar{k})=\frac{1}{k_{\perp}}\left[\begin{array}{c}
0 \\
\gamma k_{2} \\
0 \\
0 \\
k_{\perp}
\end{array}\right] \quad \epsilon_{\mu}^{3}(\bar{k})=\left[\begin{array}{l}
0 \\
1 \\
0 \\
0
\end{array}\right]
$$

with $\gamma=\frac{2 k^{+} k_{\perp}}{k_{\perp}^{2}+m^{2}}$, where we denote $\epsilon_{+}^{a}(\bar{k})$ in the first row, then $\epsilon_{-}^{a}(\bar{k})$ in the second row, then $\epsilon_{1}^{a}(\bar{k})$ in the third row and at last $\epsilon_{2}^{a}(\bar{k})$ in the fourth row. 
Open Access This article is distributed under the terms of the Creative Commons Attribution 4.0 International License (http:// creativecommons.org/licenses/by/4.0/), which permits unrestricted use, distribution, and reproduction in any medium, provided you give appropriate credit to the original author(s) and the source, provide a link to the Creative Commons license, and indicate if changes were made.

\section{References}

1. P.P. Srivastava, S.J. Brodsky, Light-front-quantized QCD in light-cone gauge: the doubly transverse gauge propagator. Phys. Rev. D 64, 045006 (2001)

2. J.A. Przeszowski, Alternate light front quantization procedure for scalar fields. Few-Body Syst. 58, 102 (2017)

3. A. Proca, Sur la théorie ondulatoire positifs et négativis. J. Phys. Radium. 7, 347-353 (1936)

4. C. Itzykson, J.-B. Zuber, Quantum Field Theory (Dover Publication Inc., Mineola, 1980)

5. J.A. Przeszowski, Lorentz symmetry for the light-front Wightman functions. Acta Phys. Pol. Proc. Suppl. B 6, 327-333 (2013)

6. M. Burkardt, Light front quantization. Adv. Nucl. Phys. 23, 1-74 (1996)

7. S.J. Brodsky, H.-C. Pauli, S. Pinsky, Quantum chromodynamics and other field theories on the light cone. Phys. Lett. C (Phys. Rep.) 301, 299-486 (1998)

8. P.A.M. Dirac, Generalized Hamiltonian dynamics. Can. J. Phys. 2, 129-148 (1950)

9. P.A.M. Dirac, Lectures on Quantum Mechanics (Yeshiva University Press, New York, 1964)

10. D.E. Soper, Massive quantum electrodynamics in the infinite-momentum frame. Phys. Rev. D 4, 1620-1625 (1971)

11. J. Przeszowski, H.W.L. Naus, A.C. Kalloniatis, Quantum electrodynamics in the light-front Weyl gauge. Phys. Rev. D 54, 5135-5147 (1996)

12. R. Soldati, M. Morara, Consistent perturbative light-front formulation of quantum electrodynamics. Phys. Rev. D 58, 105011 (1998) 